SCHWERPUNKT

\section{Megacities - ein zentraler Faktor für eine global nachhaltige Entwicklung}

\section{Einführung in den Schwerpunkt}

\section{von Helmut Lehn und Jürgen Kopfmüller, ITAS}

Urbanisierung stellt eines der zentralen Phänomene globaler Entwicklung insbesondere seit dem Zweiten Weltkrieg dar. Die Entwicklung von Megacities und von Global Cities spielt aufgrund ihrer Geschwindigkeit, ihres Ausmaßes und ihrer weltweiten Auswirkungen eine besondere Rolle. Während Megacities durch die Einwohnerzahl definiert werden, diese jedoch in der Forschungsdiskussion zwischen fünf, acht und zehn Mio. Einwohnern variiert, werden Global Cities durch ihre Funktion als einflussreiche Knoten im globalen Wirtschafts-, Finanz- und Politiknetzwerk charakterisiert. Diese Städte sind einerseits treibende Kraft des globalen Wandels, andererseits akkumulieren sich gerade hier dessen Auswirkungen. Insofern sind diese Stadttypen zugleich „Täter“ und „Opfer“ (potenziell) krisenhafter und riskanter Erscheinungsformen des globalen Wandels.

Dennoch bieten Megastädte aufgrund verschiedener Merkmale große Potenziale für die Verbesserung der menschlichen Lebensbedingungen. Zunächst sind dies ihre Größe und ihre Konzentration von Menschen unterschiedlicher Herkunft. Darüber hinaus sind es auch die Konzentration von Finanzkapital und von bedeutsamen Institutionen, die sie zu Knotenpunkten in den globalen Netzen wirtschaftlicher, politischer und auch kultureller Entwicklung machen. Sie sind dadurch Orte des ökonomischen Fortschritts, für Innovationen sowie für eine effiziente Bereitstellung von Gütern, Dienstleistungen und lebensnotwendigen Infra- strukturen. Gleichzeitig bergen sie jedoch auch erhebliche soziale und ökologische Risiken, die sich sowohl auf ihre eigene Funktionsfähigkeit als auch auf globale, nationale und regionale Entwicklungsprozesse auswirken. In dieser Chancen-Risiken-Ambivalenz kommt Megacities eine zentrale Bedeutung für die Realisierung einer global nachhaltigen Entwicklung zu.

Dabei hängt das Wohlergehen der Menschen in Metropolen und ihr effizienter, an Grundsätzen der Nachhaltigkeit orientierter Umgang mit Naturressourcen insbesondere im Kontext städtischer Infrastrukturen (Energie, Verkehr, Abfall, Wasser und Abwasser) entscheidend vom Einsatz und Management bestimmter Technologien und Techniken ab. Deshalb kommt der Abschätzung von Folgen und Nebenfolgen eingesetzter oder potenziell vorhandener Techniken eine Schlüsselrolle bei der Entwicklung von Strategien für diese Agglomerationen in Richtung von mehr Nachhaltigkeit zu.

Um die vielfältigen noch bestehenden Wissens- und Handlungsdefizite zu beheben, ist in den letzten Jahren ein eigenes internationales Forschungsfeld entstanden, in dem eine Vielzahl von Themen unter verschiedenen regionalen und disziplinären Gesichtspunkten untersucht wird. Es geht hier zum einen um Fragen zur Entstehung von Megacities und zu ihrer Rolle in regionalen, nationalen und globalen Entwicklungsprozessen. Zum anderen wird die große Bandbreite spezifischer Problemfelder behandelt und nach angemessenen Wegen zu ihrer Lösung bzw. Minderung gesucht.

In Deutschland existieren derzeit drei Forschungsinitiativen, die in diesem Forschungsfeld tätig sind: das Programm des Bundesministeriums für Bildung und Forschung (BMBF) „Megacities of Tomorrow / Future Megacities“, das Programm der Deutschen Forschungsgesellschaft (DFG) „Megacities - Megachallenge. Informal Dynamics of Global Change" sowie die Forschungsinitiative der HelmholtzGemeinschaft (HGF) „Risk Habitat Megacity“. Diese drei Initiativen und ihre Einzelprojekte arbeiten an unterschiedlichen thematischen und räumlichen Schwerpunkten. Gemeinsam ist ihnen jedoch, dass sie sich mit maßgeblichen Faktoren der Entwicklung von Megastädten und künftigen Trends befassen und dass sie konkrete umsetzungsorientierte Lösungsansät- 
ze für den Umgang mit existierenden Problemen erarbeiten.

Dieser TATuP-Schwerpunkt verfolgt im Wesentlichen zwei Ziele: Zum einen sollen Megacities in ihrer spezifischen Charakteristik, in einigen ihrer zentralen Problemlagen hinsichtlich ihrer Rolle in Prozessen des globalen Wandels und in ihrer Bedeutung für eine global nachhaltige Entwicklung beschrieben werden. Zum anderen soll ein Überblick über die diesbezüglichen Forschungsaktivitäten in Deutschland gegeben werden.

Der erste Teil des Schwerpunkts umfasst zwei Beiträge, die eine grundsätzliche Beschreibung und Einschätzung des globalen Phänomens „Megacities“ in ihren Mittelpunkt stellen: Axel Borsdorf und Martin Coy vom Geographischen Institut der Universität Innsbruck betrachten das Verhältnis von Megacities und Globalem Wandel. Globaler Wandel wird dabei umfassend als die ,kausalen Beziehungen und vielfältigen Wechselwirkungen zwischen den weltweit wirksamen Veränderungen der natürlichen und gesellschaftlichen Systeme" verstanden, wobei den Megacities sowohl die Rolle von Schauplätzen („Opfer") als auch von Motoren (,Täter") des Wandels zuzuschreiben ist. Die Autoren greifen das Beispiel Lateinamerika heraus und beschreiben als prägendes Kennzeichen des Strukturwandels der Städte Lateinamerikas den Transformationsprozess von der bipolaren (d. $h$. in einen ,armen" und einen ,reichen“" Teil getrennte) zur „fragmentierten Stadt“. Diese neue Form städtischer Entwicklung ist gekennzeichnet durch ein differenzierteres Muster armer und reicher "Zellen“. Der urbane Strukturwandel wird dabei in den Kontext eines umfassenden, in den 1970er Jahren begonnenen Transformationsprozesses gestellt, der durch eine weitgehende Öffnung für Globalisierungsprozesse und eine weitreichende Privatisierung öffentlicher Dienstleistungen geprägt ist. Die vermehrt entstehenden „barrios cerrados" und „ciudades valladas", also eingezäunte Wohngebiete vorwiegend für die Mittel- und Oberschicht, teilweise aber auch für ärmere Bevölkerungsgruppen, werden aufgrund der erheblichen Abschottungs- und Ghettoisierungstendenzen als „Gegenmodell zur traditionellen Stadt" beschrieben, weil in ihnen wichtige städtische Funktionen (wie etwa die freie Zugänglichkeit des öffentli- chem Raumes und öffentlicher Einrichtungen) erheblich eingeschränkt sind.

Rüdiger Korff und Eberhard Rothfuß von der Philosophischen Fakultät der Universität Passau betonen in ihrem Beitrag „Ambivalence of Megacities: Catastrophe or Solution?" die in der bisherigen Forschung ihrer Meinung nach unterbelichtete Funktion von Megacities als Zentren von Innovationen und Problemlösungen. Im Hinblick auf die Rolle von Megacities für eine nachhaltige Entwicklung und die dafür erforderliche „urbane Revolution“ betonen sie die herausragende Rolle städtischer Selbstorganisation. Im Unterschied zum bisherigen technologie- und expertenbasierten Urbanisierungsmodell, das durch die offenkundigen Grenzen von Steuerung, Planung und Integration gekennzeichnet ist und das die Autoren als wesentliche Ursache für die existierenden Probleme sehen, geht es hier vor allem um ein verändertes Verständnis von Governance. Es basiert auf einer verbesserten Kommunikation zwischen Bürgern und Verwaltung, Konsensorientierung, Multikulturalität und einer stärkeren Einbeziehung von Bürger-Expertise in Entscheidungsprozesse. Am Beispiel dreier Städte (je eine in Brasilien, Indien und China) skizzieren sie die Notwendigkeit, die unterschiedlichen kulturellen oder auch institutionellen Kontexte einzelner Städte in solchen Strategieänderungen zu berücksichtigen, aber auch die jeweils gleich bedeutsame Rolle der ärmsten Slumviertel für das Gelingen einer solchen selbstorganisierten, konsensualen Stadtentwicklungssteuerung einzubeziehen.

Der zweite Teil des Schwerpunkts fokussiert mit seinen beiden Beiträgen auf die Rolle von Megacities als Orte der Konzentration von Finanzkapital und von Konzernzentralen als Knotenpunkte in den globalen Netzen wirtschaftlicher und politischer Entwicklung sowie auf die teilweise erheblichen Steuerungsprobleme, die sich aus der Größe, Unübersichtlichkeit und den anzutreffenden informellen Strukturen ergeben. Im Beitrag „Die Megastädte des Südens in der Geographie globaler Finanzmärkte und weltweiter Unternehmensnetzwerke" macht Christof Parnreiter vom Geographischen Institut der Universität Hamburg deutlich, dass die Megastädte des „Südens“ sowohl auf den globalen Finanzmärkten - gemessen an den Indikatoren „Börsenkapitalisierung“ und „Aktienhandel“ als auch als Zentren weltweiter Unternehmens- 
netzwerke bisher keine wesentliche Rolle spielen. Neben den vier global dominanten Zentren New York, Tokio, London und Paris spielen in den Ländern des Südens nur Shanghai, Peking, Mumbai und São Paulo (als einzige Stadt Lateinamerikas) eine erwähnenswerte Rolle, wenn die wirtschaftliche Macht als Kriterium hierfür gewählt wird. Dies und die Rolle von beispielsweise Frankfurt oder Zürich als Nicht-Megacities im Finanzsektor zeigen eindrücklich, dass „Megacity“ nicht gleichbedeutend mit „Global City" sein muss. Am Beispiel von Mexiko-City wird anhand der Indikatoren „Außenhandelsquote“, „Ausländische Direktinvestitionen“ und „Zentralisierung von Unternehmensdiensten“" aber auch gezeigt, dass die Stadt durch das Einspeisen hochwertiger Unternehmensdienstleistungen in globale Güterketten durchaus Funktionen einer Global City übernimmt. Daran anknüpfend wird jedoch auch kritisch reflektiert, dass den Metropolen Lateinamerikas eine Art Brückenkopffunktion des „Nordens“ zukommt, die den Abfluss von Finanz- und Humankapital aus dem Süden und damit eine weitere Verschärfung einer global ungleichen Entwicklung ermöglicht.

Günter Mertins vom Geographischen Institut der Universität Marburg beschreibt in seinem Beitrag „Megacities in Lateinamerika: Informalität und Unsicherheit als zentrale Probleme von Governance und Steuerung" zunächst die Problematik der Definition von „Informalität“. Anhand seiner Analyse informeller Strukturen von Wirtschafts- und Siedlungsaktivitäten in Lateinamerika kommt er zu der Schlussfolgerung, dass informelle Prozesse zum einen aufgrund jahrzehntelanger Ignoranz und Toleranz, zum anderen wegen ihrer wichtigen, das Überleben sichernden Auffangfunktionen inzwischen - auch unter normativen Gesichtspunkten - in Lateinamerika als Realität angesehen werden müssen. Die hieraus resultierende mangelnde Steuerbarkeit der Megacities durch die klassischen formellen Strukturen wird verstärkt durch die seit den 1980er Jahren ständig zunehmende „violencia moderna", d. h. organisierte Kriminalität im großen Stil. Sie hat innerhalb der Megastädte zu Herrschaftsräumen krimineller Organisationen geführt, die dem öffentlichen Recht und der Staatsgewalt weitgehend entzogen sind und die von „mikrolokalen“" Warlords beherrscht werden. Wenig erforscht und verstanden sind dabei die Wechselwirkungen zwischen der informellen Privatisierung öffentlicher Aufgaben und informellen Beziehungsgeflechten der Oberschicht (z. B. Steuerhinterziehung) einerseits und dem fließenden Übergang von ökonomischem und wohnungssozialem Abstieg zu kriminellen informellen Tätigkeiten (z. B. Drogenhandel) und zur Mitwirkung in informellen kriminell-mafiösen Organisationen und informeller Politik mit Korruption, Tolerierung von Gewalt bzw. Straffreiheit für Täter andererseits. Eine der entscheidenden Fragen ist hier, inwieweit das Zusammenwirken von formellen und informellen Akteuren und Aktivitäten die Realisierung einer ,good governance“ eher fördert oder eher behindert und wie ein förderliches Zusammenwirken im Detail aussehen müsste.

Im dritten Teil des Schwerpunkts geht es darum, einen Überblick über die aktuell in Deutschland angelaufenen Forschungsprogramme zum Thema Megacities zu geben und diese in zwei Fällen näher vorzustellen.

Eckhart Ehlers vom Geographischen Institut der Universität Bonn gibt in seinem Beitrag „Megastädte als Herausforderung für deutsche und internationale Forschung. Ein Plädoyer für Kommunikation und Erfahrungsaustausch“" einen kurzen Überblick über die drei derzeit in Deutschland laufenden Forschungsinitiativen in diesem Feld: das BMBF-Programm „Megacities of Tomorrow/Future Megacities" mit seinen zehn Einzelprojekten, das DFG-Programms „Megacities - Megachallenge. Informal Dynamics of Global Change" mit seinen zehn Forschungsgruppen sowie die HGF-Forschungsinitiative „Risk Habitat Megacity“. Im Mittelpunkt steht seine Einschätzung, dass diese Vorhaben in ihrer Gesamtheit eine Vorreiterrolle in der internationalen Megastadtforschung übernehmen. Nach Ansicht des Autors ist eine wesentliche Voraussetzung hierfür, dass in allen drei Programmen Grundlagenforschung und Praxisbezug auf der Basis gleichberechtigter Partnerschaft zwischen ausländischen und deutschen Wissenschaftlern, sowie in inter- und transdisziplinärer Arbeitsweise gekoppelt werden. Hierauf aufbauend mahnt er einen permanenten Erfahrungsaustausch aller Projekte, den Aufbau einer gemeinsamen Datenbank und die engagierte Sichtbarkeit der Programminitiativen nicht nur bei (fach-) wissenschaftlichen, sondern auch bei Veranstaltungen politischen 
Charakters (z. B. UN-HABITAT, World Urban Forum, Weltbank) an.

Jürgen Kopfmüller und Helmut Lehn vom Institut für Technikfolgenabschätzung und Systemanalyse im Forschungszentrum Karlsruhe sowie Dirk Heinrichs, Kerstin Krellenberg und Henning Nuissl vom Helmholtzzentrum für Umweltforschung in Leipzig stellen in ihrem Beitrag die Ziele, den Untersuchungsansatz und ausgewählte Fragestellungen der Forschungsinitiative „Risk Habitat Megacity“ der HelmholtzGemeinschaft deutscher Forschungszentren (HGF) vor. Sie begründen die Auswahl des Untersuchungsraums Lateinamerika und der Fallstudie Santiago de Chile und skizzieren die konzeptionelle und analytische Architektur des Projekts, die auf einer systematischen Anwendung des Nachhaltigkeits-, des Risiko- und des Governancekonzepts auf verschiedene megacity-typische Problem- und Handlungsfelder basiert. Am Beispiel der Anwendungsfelder „Wasser" und „sozialräumliche Differenzierung" werden wesentliche im Projekt behandelte Fragestellungen und methodische Herangehensweisen skizziert. Die Autoren illustrieren damit auch die Arbeitsgruppen übergreifende Verwendung von Nachhaltigkeitsindikatoren und Szenarien.

In ihrem Beitrag „Das Beziehungsgeflecht ,Megacity / Hinterland' am Beispiel der Wasserproblematik der chinesischen Megacity Urumqi“" beschreiben Katharina Fricke, Thomas Sterr, Olaf Bubenzer und Bernhard Eitel vom Geographischen Institut der Universität Heidelberg die typischen Problemlagen großer und wachsender Agglomerationen hinsichtlich der Versorgung mit Naturgütern (hier Wasser) und der Entsorgung der in ihnen generierten unerwünschten Produkte (hier Abwasser). Urumqi, die in der Autonomen Republik Xinjiang in der Volksrepublik China gelegene und am weitesten vom Meer entfernte Großstadt der Erde, kann hierbei aufgrund ihrer "Oasenlage“" zwischen der Gurbantünggüt-Wüste im Norden und den Tianshan-Bergen im Süden sicherlich als ein Ort in geografischer Extremlage angesehen werden. Entsprechend zeigen die vorhandenen, erneuerbaren Wasserressourcen bereits heute deutliche Anzeichen von Übernutzung. Zusätzliche natürliche Wasserquellen sind nur über Ferntransporte oder durch die Nutzung von Wasserquellen möglich, die sich nicht mehr erneuern können.
Das anhaltende Bevölkerungswachstum wird bei gleichzeitig sehr dynamischer Entwicklung der Wirtschaft und dem damit verbundenem Anstieg des Lebensstandards zu einer überproportional steigenden Nachfrage nach Wasser führen, so dass sich - auch aufgrund der Auswirkungen des Klimawandels - die Schere zwischen Angebot und Nachfrage weiter öffnen wird. Dieser Umstand sowie erkennbare Nutzungsrestriktionen aufgrund nur eingeschränkt aufbereiteter Abwässer lassen ein integriertes, an Nachhaltigkeitsgrundsätzen orientiertes Wassermanagement unter Einbeziehung Effizienz fördernder Technologien dringlich erscheinen ein Erfordernis, das sich auch für viele andere Megacities stellt.

Abschließend sei noch darauf hingewiesen, dass für diesen Schwerpunkt auch ein Beitrag von den chilenischen Projektkoordinatoren der Risk-Habitat-Megacity-Initiative vorgesehen war. Er wird sich insbesondere mit der gegenwärtigen und zukünftig erwarteten Rolle von Santiago de Chile innerhalb Chiles, von Chile innerhalb Lateinamerikas sowie von Lateinamerika im globalen Kontext befassen. Vertieft werden dabei insbesondere die Bedingungen für die Realisierung einer nachhaltigen Entwicklung; damit wird dieser Beitrag wieder von konkreten Projektergebnissen zur Rolle von Megacities für globale Entwicklungsprozesse zurückführen. Leider ist es nicht gelungen, diesen Beitrag rechtzeitig zur Drucklegung fertig zu stellen. Er ist für die nächste Ausgabe dieser Zeitschrift eingeplant.

\section{Kontakt}

Dr. Helmut Lehn

Forschungszentrum Karlsruhe in der HelmholtzGemeinschaft

Institut für Technikfolgenabschätzung und Systemanalyse (ITAS)

Postfach 36 40, 76021 Karlsruhe

Tel.: +49 (0) 7247 / 82 - 3977

E-Mail: lehn@itas.fzk.de

$\langle\gg$ 